\title{
Total Power Failure in a Desflurane Vaporizer Resulting in Discontinuation of Anesthetic Delivery
}

\author{
Won K. Chee \\ Assistant Professor, Department of Anesthesiology, Montefiore Medical Center \& Albert Einstein College of Medicine, USA.
}

\begin{abstract}
An unrecognized power failure in a desflurane vaporizer is possible with a concomitant alarm battery failure. Consequent discontinuation of desflurane delivery may go unrecognized and create the potential for intraoperative awareness and inadequate depth of anesthesia. Preventive measures could include assignment of an exclusive power outlet for the desflurane vaporizer, periodic replacement and testing of the alarm battery, vigilant monitoring and documentation of desflurane levels, and even potential re-design of the vaporizer alarm system.
\end{abstract}

\section{*Corresponding Author:}

Won K. Chee, M.D., M.B.A,

Assistant Professor, Department of Anesthesiology, Montefiore Medical Center \& Albert Einstein College of Medicine, 111 East 210 $0^{\text {th }}$ Street, Bronx, New York, 10467, USA.

E-Mail: WCHEE@montefiore.org

Received: February 12, 2015

Accepted: March 13, 2015

Published: March 16, 2015

Citation: Won K. Chee (2015) Total Power Failure in a Desflurane Vaporizer Resulting in Discontinuation of Anesthetic Delivery. Int J Anesth Res. 3(2), 91-92. doi: http://dx.doi.org/10.19070/2332-2780-1500023

Copyright: Won K. Chee ${ }^{\circ} 2015$. This is an open-access article distributed under the terms of the Creative Commons Attribution License, which permits unrestricted use, distribution and reproduction in any medium, provided the original author and source are credited.

\section{Introduction}

The desflurane vaporizer (Tec 6 Plus Vaporizer by Datex-Ohmeda) requires electrical power for heating the agent to $39^{\circ} \mathrm{C}$ to establish optimal vapor pressure for delivery of anesthesia [1]. Without electrical power the vaporizer's dial will remain locked to prevent initiation of agent delivery; meanwhile, ongoing delivery of desflurane will stop within 10 seconds in the setting of power failure [2]. Such an unexpected loss of power should be recognized via an auditory alarm and visual display - both of which are powered by a 9 -volt battery incorporated in the base of the vaporizer. This case report describes a uniquely rare situation in which the discontinuation of desflurane delivery went unrecognized due to concomitant failure of the backup alarm battery.

\section{Case Description}

A healthy 35-year-old male was scheduled for open reduction and internal fixation of a right ankle fracture under general anesthesia. He denied any significant past medical history and presented with normal vital signs and an unremarkable airway. Induction of general anesthesia and endotracheal intubation were achieved with intravenous administration of propofol $(200 \mathrm{mg})$, midazolam (2 $\mathrm{mg})$, fentanyl (100 $\mathrm{mcg}$ ) and rocuronium (50 mg). Intraoperative anesthesia was maintained with an inhalational admixture of $50 \%$ nitrous oxide/oxygen and $6 \%$ desflurane. Initial skin incision resulted in a blood pressure rise from 120/70 to 159/96 and the heart rate from 82 to 125 . Despite additional fentanyl bolus (total $250 \mathrm{mcg}$ ), the blood pressure and the heart rate remained elevated and the patient was noted to be diaphoretic. Inspection of the anesthesia machine revealed an unpowered desflurane vaporizer without function of the LED light panel or LCD agent level indicator. Sevoflurane was started immediately followed by intravenous midazolam $(6 \mathrm{mg})$. Hemodynamic values returned to baseline over the next several minutes, and the remainder of the case was unremarkable. The patient was extubated and transferred to the recovery unit. An interview was conducted on postop day 1, and the patient did not report any recall or intraoperative awareness.

\section{Discussion}

Numerous reasons for vaporizer failures have been reported in the literature. Some failures are centered on the potential for electrical or mechanical errors inherent to the devices themselves, and some are a product of human error. General chamber compromise, improper mounting, overfilling, tilting, or incompatibilities with certain anesthesia machine systems have all been shown to produce inaccurate anesthetic delivery [3-8]. Unlike most common vaporizers, the desflurane vaporizer can be considered a "smarter" device - one capable of recognizing and reporting potential clinical problems as they arise. However, such features can present a double-edged sword for the clinician: a situation in which vigilance can be supplanted by electronics.

Although rare, inherent safety features of the Tec 6 Plus Vaporizer (the one used in this case) can be overridden or go unrecognized. To begin, when the vaporizer is powered up, an internal system check is supposed to indicate a low battery level through 
an LED display. This is not fail-safe. For instance, if the alarm battery depletes beyond the alarm threshold charge following activation of the vaporizer, detection of the vaporizer power failure will depend solely on the clinician. That is, the clinician must recognize the loss of the LED display, or notice the decreasing exhaled end-tidal agent concentration on the anesthesia monitor.

In another scenario involving a normally functioning vaporizer, if desflurane is being delivered while the main power supply is interrupted, delivery of the gas should stop within 10 seconds. This unexpected loss of power should then become immediately apparent by the auditory alarm and LED error display, which is activated by a 9-volt battery incorporated in the base of the vaporizer. Obviously, if the battery is fully depleted, then furtherdue diligence is placed on the clinician to avert complications. (Of note, the alarm battery is not designed to resume desflurane delivery).

Finally, although the vaporizer itself requires very little power, a failure of the desflurane vaporizer may be possible from sharing the power source with other equipment [9]. For example, multioutlet cords are frequently used to supply electric power to Anesthesia Information Management Systems attached to the anesthesia machine. When the desflurane vaporizer is also connected to such a multi-outlet strip, exceeding the power limit or an electrical surge can cause a silent shutdown. Once again, the 9-volt battery and clinician are on the hook.

In closing, minimal measures to prevent unrecognized vaporizer failure should include:
1. An assignment of an unshared power outlet for the desflurane vaporizer

2. Annual replacement and periodic testing of the battery and power failure alarm in the vaporizer

3. Vigilant intra operative monitoring of the vapor agent levels on the monitors

Although beyond the breadth and scope of this discussion; one could also imagine potential vaporizer design modifications that could further mitigate this scenario.

\section{References}

[1]. Andrews JJ, Johnston RV Jr (1993) The new Tec 6 desflurane vaporizer. AnesthAnalg 76(6): 1338-41.

[2]. (2001) Operation and Maintenance Manual for Tec 6 Plus vaporizer, DatexOhmeda Division, Instrumentarium Corp, Finland.

[3]. Ghai B, Makkar JK (2005) An unusal cause of faulty Tec 7 Vaporizer. AnesthAnalg 101(6): 1890-1891.

[4]. MacLeod DM, McEvoy L, Walker D (2002) Report of vaporizer malfunction. Anaesthesia 57(3): 299-300.

[5]. Chambers JC, Hough MB (2005) Awareness hazard using a Tec 6 vaporiser. Anaesthesia 60(9): 942.

[6]. Fernando PM, Peck DJ (2001) An uncommon cause of vaporiser failure. Anaesthesia 56:1009-10.

[7]. Atlee JL (2007) Complications in Anesthesia (2nd edtn), Equipment and Monitoring. Elsevior Health Sciences, Philadelphia, 531-32.

[8]. Kimatian SJ (2002) With technology comes responsibility: intraoperative failure of an anesthetic vaporizer. Anesthesiology 96(6): 1533-4.

[9]. Goodman EJ, Hudson IM, Douglas L (1999) Desflurane Vaporizer Uses Minimal Electricity. AnesthAnalg 88(5): 1189. 